\title{
Phasic alertness indicated by simple motor reaction time in late childhood: The effect of age and sex
}

\author{
Rudolf Psotta ${ }^{1 凶}$, Josef Kraus², Milada Krejčí ${ }^{3}$, and Grzegorz Juras ${ }^{4}$ \\ ${ }^{1}$ Department of Natural Sciences in Kinanthropology, Faculty of Physical Culture, Palacký University Olomouc, Olomouc, Czech \\ Republic; ' ${ }^{2}$ Clinics of Pediatric Neurology, Faculty Hospital Motol, 2nd Faculty of Medicine, Charles University, Prague, Czech Repubic; \\ ${ }^{3}$ College of Physical Education and Sport PALESTRA, Prague, Czech Republic; and ${ }^{4}$ Department of Human Motorics, Jerzy Kukuczka \\ Academy of Physical Education in Katowice, Katowice, Poland
}

\begin{abstract}
Background: In contrast to strongly established views on the development of tonic, top-down controlled alertness, a small number of neurobehavioral and neurophysiological studies deal with the developmental trajectory of exogenously driven phasic alertness during childhood. Objective: The current study aimed to examine the age and sex effects on phasic alerting during late childhood, and to analyse the degree of association between tonic and phasic alertness at the behavioural level. Methods: Three age groups of typically developing children, aged 10, 11 and 12 years ( $N=59,27$ boys, 32 girls) performed a computer-based simple hand-eye reaction test of alertness that involved 28 trials with and 28 trials without a warning signal. Results: The results showed a moderate decrease of mean reaction time in both the alert and non-alert conditions with age. However, the alert effect calculated as the difference in reaction times achieved in alert and non-alert conditions was not affected by age and sex. Conclusions: In contrast to previous suggestions on possible continuing improvement in phasic alertness during late childhood, the current study suggested that the neurocognitive function of transient enhancement in attentional alertness (phasic alertness) does not change and it is stabilized in this developmental period. In addition, this function does not differ between males and females in childhood.
\end{abstract}

Keywords: development, attention, test, children

\section{Introduction}

The current conceptualization of attention differentiates between the alerting, orienting and executive networks which are functionally independent and responsible for the different cognitive processes related to attention (Petersen $\&$ Posner, 2012; Posner, 2012). The alerting network is responsible for the attentional alertness that signifies the intensity dimension of attention. Attentional alertness is the state of response readiness that involves high sensitivity to incoming stimuli (Petersen \& Posner, 2012; Posner, 2008), preparedness for information processing and response execution (Keehn et al., 2013).

The basic mode of alertness called tonic (intrinsic) alertness is endogenously controlled responsiveness to external stimuli, in the absence of an external cue in a topdown manner, and refers to sustained attention or vigilance (Keehn et al., 2013). Tonic alertness changes over the course of the day (diurnal and circadian rhythm) including slow fluctuations over hours or minutes (Posner, 2008; Shalev et al., 2016). Based on simple mean reaction time, improvement in tonic alertness across childhood and adolescence has been reported (Dykiert et al., 2012; Sobeh \& Spijkers, 2013).
However, intrinsic alertness can temporarily increase for milliseconds or seconds to a target stimulus (task) when triggered from an external warning or an unexpected or sudden cue that closely precedes a target stimulus (Petersen $\&$ Posner 2012; Shalev et al., 2016). This transient, phasic alertness consists in exogenously driven increased activation of the alerting network in a bottom-up manner when induced by a transient sensory input such as short sound, change in lighting, etc. (Keehn et al., 2013; Sturm \& Willmes, 2001). Researchers have thought that neural mechanisms that are probably responsible for the warning-cue effect on shortening of a response, concern facilitation of early perceptual encoding (Correa et al., 2006), increasing visual processing speed, decreasing the threshold of conscious perception (Petersen et al., 2017), and/or motor programming/execution (Fischer et al., 2013). Operationally, phasic alertness is defined as the ability to decrease reaction time (RT) when the target stimulus is preceded $(-100$ to $2000 \mathrm{~ms})$ by a non-informative warning cue (Schuhfried, 2011; Zimmermann \& Fimm, 2002). The alerting effect is expressed by a subtraction of RT achieved in a no-cue condition and cue condition in the same reaction task (alerting score; Keehn et al., 2013; Schuhfried, 2011).

$\triangle$ Corresponding author: Rudolf Psotta, e-mail rudolf.psotta@upol.cz, ORCID® record https://orcid.org/0000-0001-5563-8974

Article history: Received June 30 2021, Accepted November 2 2021, Published November 152021

Copyright: @ 2021 The Author(s). Published by Palacký University Olomouc. This is an open access article distributed under the terms of the Creative Commons Attribution License (https://creativecommons.org/licenses/by/4.0/), which permits unrestricted use, distribution, and reproduction in any medium, provided the original author and source are credited. This license does not cover any third-party material that may appear with permission in the article. 
Including phasic alertness in a neuropsychological assessment could be useful for the diagnostic process when children with attention deficit hyperactivity disorder (ADHD) and autism spectrum disorder (ASD) have shown lower ability to process information accurately in the alert state evoked by a warning signal (Cao et al., 2008; Keehn et al., 2013). It should be noted that the neural basis for phasic alertness differs from that of tonic alertness. Tonic alertness relates to activity in the right-sided fronto-parietothalamic network (Sturm et al., 2004), while phasic alertness relates, in addition, to activity in the left-hemisphere frontal and parietal structures (Sturm \& Willmes, 2001). Thus, assessment of phasic alertness using a reaction task with both no-cue and cue trials could show the functioning of the aforementioned neural structures.

However, compared to the tonic and other aspects of attention, understanding the development of phasic alertness is scarce. Developmental improvement in phasic alerting was reported in children aged 5 up to 12 years (Lewis et al., 2018; Mezzacappa, 2004; Mullane et al., 2016). On the other hand, the study by Rueda et al. (2004) suggested the advantage of a warning cue to speed up a response to a target stimulus stabilizes by $8-9$ years of age. This finding corresponds to the results of neurophysiological investigation of the activity of the brain structures that indicated development of phasic attentional mechanisms to 7-8 years, with possible functioning at adult levels (for a review, see Keehn et al., 2013).

However, the aforementioned studies with somewhat equivocal findings used the Attention Network Test (ANT) to investigate of functioning of all three attentional networks including phasic alerting. The ANT is a two-choice reaction (flanker) task that involves responses to alternative target stimuli, and different warning cues alternately preceding target stimuli. The flanker task involved in the ANT taps executive attention. Thus, a participant might switch to top-down executive control or increase intrinsic alertness throughout the performance of the test rather than respond automatically in a bottom-up manner to prevent a decrease in response accuracy. It is a little different from alerting that hastens the speed of responding at the cost of decreased accuracy (Lawrence \& Klein, 2013; Mullane et al., 2016). Thus, indices on the maturation of phasic attention might be specific to the cognitive task used.

To the best of our knowledge, only two studies investigated developmental changes of phasic alertness in children with the use of the simple reaction task. The first study (Drechsler et al., 2005) showed a significant decrease in tonic alertness indicated by simple RTs but without significant changes in alert effect during 2.6 years in typically developing children aged 8-12 years. The second study (Querne et al., 2009) suggested stabilization of the function of phasic alertness already at the age of 8-9 years. These findings are dissimilar from the study by Konrad et al. (2005) that reported a smaller alerting effect both at the behavioural level, (i.e., via the performance of a perceptual-cognitive task), and at the neurophysiological level - concurrent reduction in activation of right-sided frontal-midbrain regions during alerting in children, as compared to adults.

The present equivocal findings on the developmental trajectory of phasic alertness could be partly for a considerably unbalanced portion of girls and boys within a sample involved in half of the aforementioned studies (Drechsler et al., 2005; Lewis et al., 2018; Mezzacappa, 2004). Interestingly, possible sex differences in the maturation of phasic alertness have not yet been studied. Significant sex differences in simple RT indicating tonic alertness have been reported for children aged from 4 years to 17 -year-old adolescents, with faster responses in males (Brocki \& Bohlin, 2004; Dykiert et al., 2012; Sobeh \& Spijkers, 2013). But the opposite findings were also reported, concretely lower attentional performance in tests of tonic alertness, distractibility and inhibition control in 6-10-year-old boys as compared to girls (Tremolada et al., 2019). However, the sex difference in SRT in children is still not clearly explained. The sex hormones that may contribute to sex differences in SRT in adults (Stenbaek et al., 2016) come more into play at puberty (Deary \& Der, 2005). However, higher testosterone and estradiol concentrations have been found in males as compared to females since the age of 11 years (Quaiser-Pohl et al., 2016; Yao et al., 2019). Even, significantly higher salivary testosterone concentrations were proved in boys already from 6 years of age (Ostatníková et al., 2002).

Above the mentioned notes suggest it is unclear whether the development trajectory of phasic alertness correlates to or differs from the maturation of tonic alertness, and whether it is liable to sexual dimorphism in the developmental transition from childhood to adolescence, i.e., in late childhood. Therefore, the aim of the current study was to examine the age and sex effects on phasic alertness in typically developing children aged 10 to 12 years and analyse the association between tonic and phasic alertness at the behavioural level at this age period. It has been suggested that the neural structures associated with intrinsic (tonic) attention, i.e., the right-hemisphere frontoparietal-thalamic-brainstem network, is also involved in exogenously driven increased attentional activation induced from transient sensory input (Clemens et al., 2013; Keehn et al., 2013). Therefore, we hypothesized that phasic alertness continues in development during late childhood (10-12 years) and maturation of this function may differ between some girls and boys.

\section{Methods}

\section{Participants}

Three age groups of typically developing children $(N=59)$ participated in the study: 10-year-olds ( 9 boys, 9 girls), age $126.6 \pm 3.2$ months, 11-year-olds (9 boys, 11 girls) age $136.9 \pm 3.1$ months, and 12-year-olds (9 boys, 12 girls), age $148.8 \pm 3.3$ months. The children were recruited from three public mainstream schools of three different regions. All these schools provided education according to the Framework Educational Program for Elementary Schools. The exclusion criteria were neurodevelopmental disorders 
according to the Diagnostic and Statistical Manual of Mental Disorders (5th ed.; American Psychiatric Association, 2013) such as intellectual disabilities, communication disorders, ADHD, ASD, specific learning disorder, and movement disorders, further, physical and sensory disabilities, conduct, psychotic, behavioural and neurological disorders, medication affecting attention, chronic and acute illness. On the basis of knowledge of the diagnoses of children and interviews with parents, school psychologists selected the children according to the criteria. The school psychologists were not aware of the hypotheses. Of the potential participant pool $(N=187) 39$ children were excluded according to the exclusion criteria (see above). The final sample $(N=59)$ included those children who attended their school on the days of measurement, and their parents or legal guardians handed in the written informed consent. The study was approved by the ethics committee of the Faculty of Physical Culture, Palacký University Olomouc (protocol number 31/2018).

An a priori power analysis showed that 59 participants would be sufficient to identify a significant effect of the two independent variables (age and sex) with a between-subject design with a power $(1-\beta)$ of .80 , effect size $f$ of .50 and an $\alpha$ of .05 (Faul \& Erdfelder, 1992).

\section{Neuropsychological test of attentional alertness}

Both modes of alertness were assessed by using a computerbased hand-eye simple reaction test of tonic and phasic alertness (Alertness test), version 31 of the Vienna Test System (VTS; Schuhfried, 2011). This test is the standardised exam of attentional alertness developed for cognitive and clinical psychology (Schuhfried, 2011). In this test, 56 target stimuli in the form of a yellow circle of a diameter of 3 $\mathrm{cm}$ were presented in the centre of a black background of a computer screen in the test phase. In the first 28 reaction runs, the target stimulus was presented without a cue (noalert trials). In the second half of the test, $1000 \mathrm{~ms}$ before the appearance of the target stimulus, an acoustic warning signal (stereo sound, a frequency of $2000 \mathrm{~Hz}$ ) lasting 500 ms was given (alert trials, see Figure 1).

From the initial position of the index finger of the preferred hand on the home button (diameter of $2 \mathrm{~cm}$ ), the participant reacted to the target stimulus as quickly as possible by pressing the rectangular black (response) button $(5.5 \times 1.5 \mathrm{~cm})$ on the operating panel. After the response to a target stimulus, the responder placed their index finger back on the home button. The home button was located below the response button with their near edges at a distance of $45 \mathrm{~mm}$. The RT was measured as the interval

Figure 1 Schematic diagram of the Alertness test

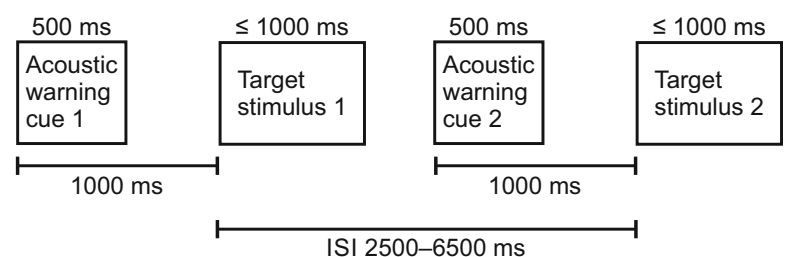

between the moment of appearance of the target stimulus and the moment the index finger left the home button. The display of the target stimulus remained until a response was detected to a maximum of $1000 \mathrm{~ms}$. Stimuli were presented in inter-stimulus intervals (ISI) from $2500 \mathrm{~ms}$ to $6500 \mathrm{~ms}$. Reliability of the Alertness test of VTS was reported as $r=.923$ and .965 for RT in alert and no-alert trials respectively (Schuhfried, 2011).

\section{Procedure}

Each participant completed the alertness test at his/her school under administration by an experimenter. Each test was conducted in a quiet room and at the same time of day, between 8-11 a.m. Each participant performed the alertness test while sitting comfortably at a table on a chair, with his/her elbows/arms resting on the table. The height of the chair was adjusted for each child so that the knee angle was approx. $90^{\circ}$. In front of the child, the VTS universal response panel with the buttons was located $5 \mathrm{~cm}$ from the edge of the table. The IBM compatible laptop computer with an Intel i7 processor and a screen of 15 inches, 1920 by 1080 pixel screen resolution, was located close behind the response panel. The participant viewed the screen from a distance of $52 \mathrm{~cm}$.

The tester was sitting next to the participant. At the beginning of the test session, the tester provided the instructions including the task goal (see above) and a very short description of the procedure. Participant began the practice block when it was obvious that he/she understood the basic instruction. Then, the participant was provided with the computer-driven practice of the test task accompanied by written instructions on the screen. During the practice, the child responded to 10 target stimuli of which 5 stimuli were preceded by the warning tone. The test computer program provided the child with visual feedback if he/she committed some error, and the experimenter also encouraged the child. After the practice block, the experimenter asked the child whether he/she fully understood the task. There was a 90 -sec break between the practice phase and the test phase. Then the child performed test trials being provided with no feedback or encouragement. The total duration of the entire session lasted around $8 \mathrm{~min}$. The tester was fully graduated in psychology and skilled and before testing, he/ she underwent repeated training of the use of the Alertness test of VTS.

\section{Neuropsychological variables}

The following neuropsychological measures were assessed: mean RT in non-alert trials as a measure of tonic alertness $\left(\mathrm{RT}_{\mathrm{NoAl}}\right)$; mean $\mathrm{RT}$ in alert trials as a measure of phasic alertness $\left(\mathrm{RT}_{\mathrm{Al}}\right)$; the alerting score calculated as the difference between mean RT in no-alert and alert trials (AS) - a measure of phasic alerting effect $\left(\mathrm{RT}_{\mathrm{NoAl}}-\mathrm{RT}_{\mathrm{Al}}\right)$; intraindividual standard deviation of RTs as a measure of the intraindividual variability of RTs in non-alert trials (ISD of $\mathrm{RT}_{\mathrm{NoAl}}$ ) and in alert trials (ISD of $\mathrm{RT}_{\mathrm{Al}}$ ). The quality of motor responses was assessed by the number of omission errors defined as the number of trials in which the 
participant did not respond within the $1000 \mathrm{~ms}$ duration of the target stimulus.

\section{Check on the execution of the test}

Test software checks incorrect responses, concretely: (i) concurrent pressing of the home button and the response button with two different fingers; (ii) the use of a finger other than the index finger to press the response button; indicated by the interval $<50 \mathrm{~ms}$ between taking the index finger off the home button and pressing the response button; (iii) taking the finger off the home button before the appearance of the stimulus. If the participant committed more than three errors, the test was stopped.

\section{Check on potential confounding variables}

We considered the following variables that might affect the performance of the test: (i) the preferred hand used by a child to complete the test task (right $=0$, left $=1$ ); (ii) a current mental status: a participant was asked about it ("well or as usual" = 0, "sleepy/tired"= 1); (iii) learning ability of the child; class teachers classified participants as "very good" = 1, "average" = 2 or "below-average" = 3 .

\section{Statistical analyses}

Reaction times shorter than $100 \mathrm{~ms}$ were excluded from the RT analysis. The Shapiro-Wilk test of normality showed a significant difference from the normal Gaussian distribution of mean $\mathrm{RT}_{\mathrm{NoAl}}$ and ISD of RTs in both non-alert and alert conditions. Therefore, the RT data were normalized using the Box-Cox transformation and put into the statistical analysis. All the measures were analysed in 3 (age: 10, 11, 12 years) $\times 2$ (sex: male, female) analysis of variance (ANOVA). The Levene's test of equality of variances showed that the assumption of homogeneity was not violated. The Bonferonni test adjustments were made for all post-hoc comparisons. A level of $\alpha=.05$ was set for all tests. Effect sizes were analysed using partial eta squared $\left(\eta_{\mathrm{p}}^{2}\right)$ with an interpretation of $\eta_{\mathrm{p}}^{2}=.01,=.06$, and .14 as small, medium, and large effects, respectively.

The mean number of omission errors was expressed as a percentage of all trials in alert and non-alert conditions, respectively. The participants made a very small number of omission errors, therefore no analyses of this variable were conducted.

The Kruskal-Wallis test or the Mann-Whitney $U$-test were used to analyse the effect of the potential confounding variables on the RT measures $(\alpha=.05)$.

To analyse the degree of determination of phasic alertness by the level of tonic alertness at the behavioural level, a simple regression analysis was carried out, in which mean RT in the alert condition was the dependent variable and mean RT in non-alert condition was the independent variable $(\alpha=.05)$. All data analyses were conducted with the SPSS program (Version 24; IBM, Armonk, NJ, USA).

\section{Results}

RT measures in the alert condition - phasic alertness Figure 2 shows moderate decreases of RTs in the alert condition $\left(\mathrm{RT}_{\mathrm{A}}\right)$ from 10 to 12 years of age. However, the results of ANOVA on $\mathrm{RT}_{\mathrm{Al}}$, ISD of $\mathrm{RT}_{\mathrm{Al}}$, and the variable of alert effect AS showed no significant effect of age, sex and the age $\mathrm{x}$ sex interaction (Table 1). Although non-significance was found, we conducted the post-hoc tests to reveal possible tendencies. The alert effect AS as the indicator of phasic alertness was significantly lower in 11-year-olds compared to 10 -year-olds $(p=.023)$ and higher than in 12-year-olds $(p=.044)$.

\section{RT measures in the non-alert condition - tonic alertness}

Figures 2 and 3 show progressive decreases of RTs and the intraindividual variability of RTs in non-alert trials $\left(\mathrm{RT}_{\text {NoAl }}\right.$

Figure 2 Mean reaction time (RT) as the function of alert in the alert and non-alert condition

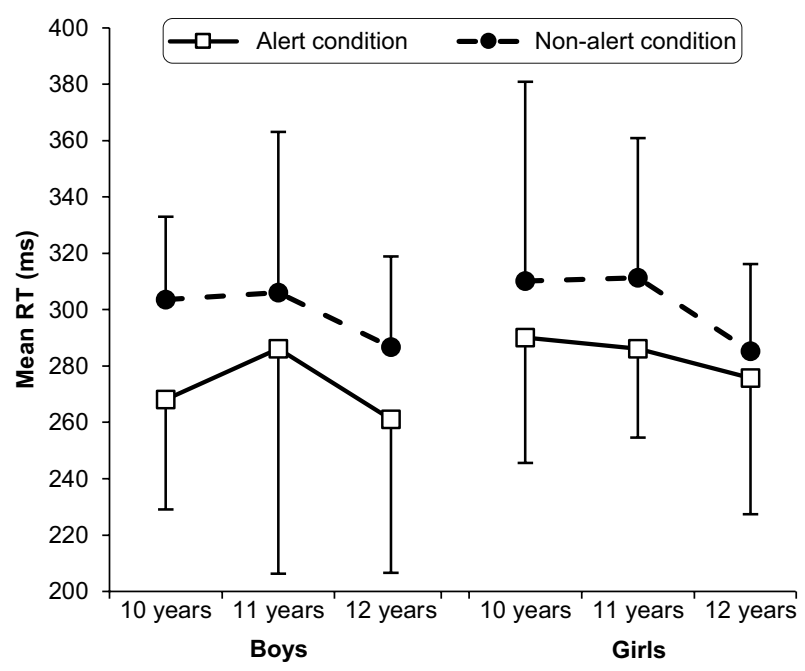

Figure 3 Intraindividual reaction time (RT) variability indicated by $S D$ of RTs as a function of alert in the alert and non-alert condition

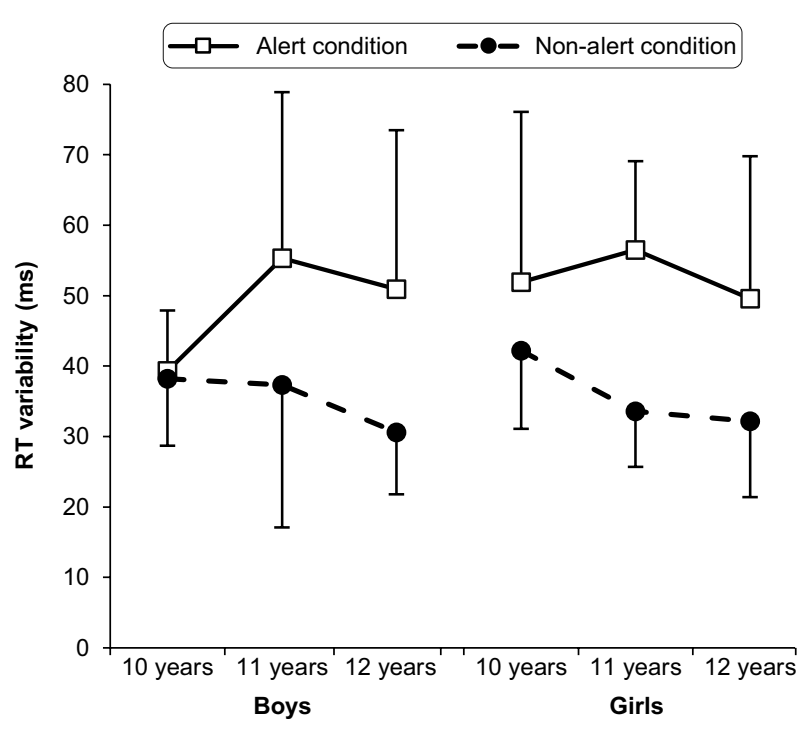


Table 1 Means, standard deviations, and the results of the analysis of variance for the reaction time (RT) measures in the non-alert condition and non-alert condition for particular ages and sex groups

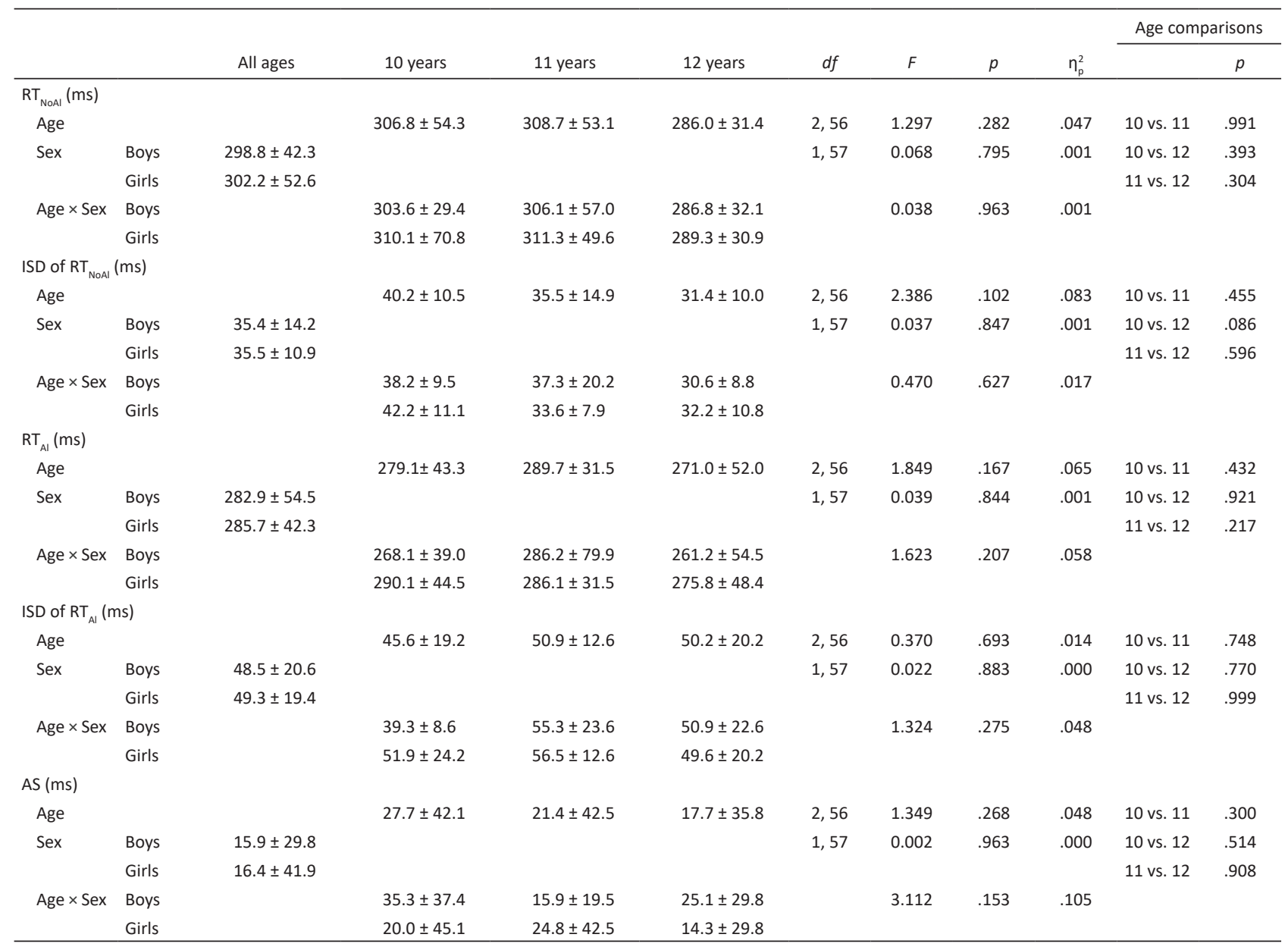

Note. $\mathrm{RT}_{\text {NoAA }}, \mathrm{RT}_{\mathrm{Al}}=$ mean reaction time in the non-alert trials and alert trials, respectively; ISD = intraindividual standard deviation; $\mathrm{AS}=$ difference between mean $\mathrm{RT}$ in no-alert and alert trials.

and ISD of $\mathrm{RT}_{\mathrm{NoAl}}$ ) from 10 to 12 years of age. However, no significant age and sex effects and interaction age $\times$ sex were found for these two variables (Table 1). Table 2 presents the number of omissions committed in the alert and non-alert conditions.

\section{Linear regression analysis}

Linear regression analysis showed that mean $\mathrm{RT}_{\text {NoAl }}$ accounted for $44.9 \%$ of the variation in the mean $\mathrm{RT}_{\mathrm{Al}}$. The regression equation for prediction of mean $\mathrm{RT}_{\mathrm{Al}}$ was:

$$
\mathrm{RT}_{\mathrm{Al}}(\mathrm{ms})=57.80+0.75 * \mathrm{RT}_{\mathrm{NoAl}}(\mathrm{ms})
$$

with the coefficient of determination $R^{2}=.449$ ( $p<.001$, $F(1,57)=46.418), r=.670$, the standard error of estimation $=41.0 \mathrm{~ms}$, corresponding to $14.4 \%$.

Check on the execution of the test

No participant committed more than three errors defined above in the Method (see Check on the execution of the test).

\section{Check on potential confounding variables}

One-way ANOVAs showed that the preferred hand, current mental status and learning ability were not significant
Table 2 The percentage of omissions and correct responses committed in the alert and non-alert condition

\begin{tabular}{lccc}
\hline & 10 years & 11 years & 12 years \\
\hline Omissions (\%) & & & \\
$\quad$ Alert condition & $0.57 \pm 1.33$ & 0 & $0.17 \pm 0.76$ \\
$\quad$ Non-alert condition & $0.40 \pm 1.12$ & $0.68 \pm 1.40$ & $0.17 \pm 0.76$ \\
Correct responses (\%) & & & \\
$\quad$ Alert condition & $99.0 \pm 1.99$ & $100.00 \pm 0.00$ & $99.15 \pm 1.52$ \\
$\quad$ Non-alert condition & $99.0 \pm 1.60$ & $99.32 \pm 1.40$ & $99.32 \pm 3.04$ \\
\hline
\end{tabular}

factors of the neuropsychological measures, $p>.05$; $\eta_{\mathrm{p}}^{2}=.000-.048, .000-.039$ and $.000-.053$, respectively.

\section{Discussion}

The goal of the present study was to examine the development of phasic alertness in typically developing children during late childhood, and concurrently, whether phasic alertness may be influenced by sex. The results showed that phasic alertness is not still changing markedly at this age period. There is, rather, a tendency for the alerting effect 
on a motor response to a visual stimulus in a simple detection task to decrease (from $27.7 \mathrm{~ms}$ to $17.7 \mathrm{~ms}$ from 10to 12 -year-old children, Table 1 ), while there is a gradual improvement in tonic alertness during late childhood. One explanation for the reduction of alert effect might be that older children may better rely on their intrinsic, endogenously, top-down controlled alertness (Lin et al., 1999) which continues to develop throughout the whole of childhood (Sobeh \& Spijkers, 2013) into adolescence (Dykiert et al., 2012), and they can use this more advanced ability to exert the cognitive control of attention gained from the maturation of the prefrontal cortex (Casey et al., 2000). In contrast, in younger children, top-down control of intrinsic alertness is not developed sufficiently, and they may have difficulty in maintaining the required alert state without a cue (Ishigami \& Klein, 2015; Posner, 2008). Thus a warning cue can evoke an increase in attentional alertness and attract their attention to a task more easily. Thus, in contrast to the suggestion on continuing maturation of the function of phasic alertness into adolescence (e.g., Konrad et al., 2005; Rueda et al., 2004; Waszak et al., 2010), the current study supports the suggestion that the capacity of the alerting attentional network for the transient increase in neural activation over a basic level of tonic alertness is maintained stably or even slightly decreases during late childhood.

The interesting finding of the current study is that not all children were able to speed up their responses in the alert condition when the proportion of children who benefited from the warning signal was $67 \%, 70 \%$ and $76 \%$ in the age of 10, 11 and 12 years, respectively. The previous studies reported the positive effect of a warning cue on RTs on average in children (Drechsler et al., 2005; Lewis et al., 2018; Mullane et al., 2016; Querne et al., 2009), however without providing information on how many individuals demonstrated no advantage from the warning cue. No alert effect of a warning signal on the shortening of the motor response found in $24 \%$ to $33 \%$ of children in the current study may suggest that some children may prefer inhibition of inappropriate responses to a warning cue, likely as compensation of insufficiently matured distinguishing between cues going in a very rapid sequence. Our study may indicate the inter-individual developmental variations of phasic alerting across childhood. These inter-individual variations of phasic attention could be attributed to neural heritability when age-correlated heritability was estimated at $40 \%$ for mean RT (Finkel \& McGue, 2007).

We also assumed that the variability of RTs achieved in the alert condition might be a more sensitive measure of maturation of phasic alertness than the alert score. It has been thought that higher intra-individual variability of RTs indicates a deficit in the regulation of energetic state and sustained attention (Andreou et al., 2007), whereas slow mean RT indicates rather difficulties in basic information processing (Collins \& Long, 1996). In the current study, we did not find any improvement in the intra-individual variability of RTs in the alert condition with age, in contrast to the observed tendency to reduce the variability of motor response in the non-alert condition with age. These results also support the stabilization of phasic alertness during late childhood.

In addition to the alert score, researchers also considered the omission errors committed in a reaction test to judge the maturation of phasic alertness. In the current study, we observed an ongoing decrease in omission errors up to the age of 12 years in the alert condition (Table 2). This finding is different from the study by Querne et al. (2009) that reported omission errors in alert trials of a simple RT test in 6-year-old children but not yet in children aged 7-10 years. It should be noted that we used the RT test with a maximal duration of $1000 \mathrm{~ms}$ of the target stimulus exposition, in contrast to $1500 \mathrm{~ms}$ used by Querne et al. (2009). It seems that the simple reaction test of alertness with a shorter exposure of stimuli might be the more sensitive measure to reveal developmental changes in phasic alertness across childhood.

To our best knowledge, no previous study has analysed the effect of sex on phasic alertness. The current study suggested that the neurocognitive function for transient enhancement in attention may not differ between males and females in childhood. We also found a very similar percentage of boys and girls, $74.1 \%$ and $71.9 \%$, respectively, who were able to use a warning cue to respond in a shorter time. In the context of the aforementioned suggestion, these results suggest an inter-individual variation rather than a sexual variation of functioning of the alerting network in children.

A limitation of the present study may be that we examined the age changes in phasic alertness in a smaller sample size and using a cross-sectional design, not via a longitudinal one. Secondly, the age range of the sample was relatively small. Another possible limitation of the present study might be the lower attractiveness of the VTS alertness test for the children tested. Rueda et al. (2004) reported that children work better in the child version of the ANT associated with a story (e.g., feeding fish via a response) and with providing feedback for each correct response (target fish blowing bubbles) as compared to the adult version of the ANT. Further, due to the fixed intervals between the auditory warning signal and the visual stimulus involved in the alertness test, an expectancy effect could be considered. However, there was reported a very little effect of the fixed foreperiods on performance (Shieh et al., 1997).

\section{Conclusions}

In contrast to previous suggestions on possible continuing improvement in phasic alertness during late childhood, the current study suggested that the neurocognitive function of transient enhancement in attentional alertness (phasic alertness) does not change and stabilizes between 10 and 12 years of age. In addition, this function does not differ between males and females in childhood. This both developmental stabilization and no significant differences between sexes in phasic alertness differ from the generally recognized continuing maturation of intrinsic attention during childhood and adolescents. The results of linear 
regression analysis suggested functional specificity of the phasic alertness from tonic alertness.

\section{Acknowledgments}

The study was funded by the Czech Science Foundation (grant 19-18787S).

The authors would like to acknowledge and thank the children, families, school managements, class teachers and school psychologists who participated in this study.

\section{Conflict of interest}

The authors report no conflict of interest.

\section{References}

American Psychiatric Association. (2013). Diagnostic and statistical manual of mental disorders (5th ed.). https://doi.org/10.1176/appi.books.9780890425596

Andreou, P., Neale, B. M., Chen, W., Christiansen, H., Gabriels, I., Heise, A., Meidad, S., Muller, U. C., Uebel, H., Banaschewski, T., Manor, I., Oades, R., Roeyers, H., Rothenberger, A., Sham, P. A. K., Steinhausen, H.-C., Asherson, P., \& Kuntsi, J. (2007). Reaction time performance in ADHD: Improvement under fast-incentive condition and familial effects. Psychological Medicine, 37(12), 1703-1715. https://doi.org/10.1017/s0033291707000815

Brocki, K., \& Bohlin, G. (2004). Executive functions in children aged 6 to 13: A dimensional and developmental study. Developmental Neuropsychology, 26(2), 571-593. https://doi.org/10.1207/s15326942dn2602 3

Cao, Q., Zang, Y., Zhu, C., Cao, X., Sun, L., Zhou, X., \& Wang, Y. (2008). Alerting deficits in children with attention deficit/hyperactivity disorder: Eventrelated fMRI evidence. Brain Research, 1219, 159-168. https://doi.org/10.1016/i. brainres.2008.04.028

Casey, B. J., Giedd, J. N., \& Thomas, K. M. (2000). Structural and functional brain development and its relation to cognitive development. Biological Psychology, 54(1-3), 241-257. https://doi.org/10.1016/s0301-0511(00)00058-2

Clemens, B., Zvyagintsev, M., Sack, A. T., Heinecke, A., Wilmes, K., \& Sturm, W. (2013). Comparison of fMRI activation patterns for test and training procedures of alertness and focused attention. Restorative Neurology and Neuroscience, 31(3), 311-336. https://doi.org/10.3233/RNN-120266

Collins, L. F., \& Long, C. J. (1996). Visual reaction time and its relationship to neuropsychological test performance. Archives of Clinical Neuropsychology, 11(7), 613-623. https://doi.org/10.1016/0887-6177(97)81255-3

Correa, A., Lupiáñez, J., Madrid, E., \& Tudela, P. (2006). Temporal attention enhances early visual processing: A review and new evidence from eventrelated potentials. Brain Research, 1076(1), 116-128. https://doi.org/10.1016/i. brainres.2005.11.074

Deary, I. J., \& Der, G. (2005). Reaction time, age, and cognitive ability: Longitudinal findings from age 16 to 63 years in representative population samples. Aging, Neuropsychology, and Cognition, 12(2), 187-215. https://doi. org/10.1080/13825580590969235

Drechsler, R., Brandeis, D., Földényi, M., Imhof, K., \& Steinhausen, H. C. (2005). The course of neuropsychological functions in children with attention deficit hyperactivity disorder from late childhood to early adolescence. Journal of Child Psychology and Psychiatry, 46(8), 824-836. https://doi. org/10.1111/i.1469-7610.2004.00384.x

Dykiert, D., Der, G., Starr, J. M., \& Deary, I. J. (2012). Sex difference in reaction time and intraindividual variability across the life span. Developmental Psychology, 48(5), 1262-1276. https://doi.org/10.1037/a0027550

Faul, F., \& Erdfelder, E. (1992). GPower: A priori, posthoc, and compromise power analyses for MS-DOS [Computer software]. Bonn University.

Finkel, D., \& McGue, M. (2007). Genetic and environmental influences in intraindividual variability in reaction time. Experimental Aging Research, 33(1), 13-35. https://doi.org/10.1080/03610730601006222

Fischer, R., Plessow, F., \& Kiesel, A. (2013). The effects of alerting signals in masked priming. Frontiers in Psychology, 4, Article 448. https://doi.org/10.3389/ fpsyg. 2013.00448

Ishigami, Y., \& Klein, R. M. (2015). Repeated measurement of the components of attention with young children using the attention network test: Stability, isolability, robustness, and reliability. Journal of Cognition and Development, 16(1), 144-159. https://doi.org/10.1080/15248372.2013.803971

Keehn, B., Müller, R. A., \& Townsend, J. (2013). Atypical attentional networks and the emergence of autism. Neuroscience \& Biobehavioral Reviews, 37(2), 164-183. https://doi.org/10.1016/i.neubiorev.2012.11.014
Konrad, K., Neufang, S., Thiel, C. M., Specht, K., Hanisch, C., Fan, J., HerpetzDahlmann, B., \& Fink, G. R. (2005). Development of attentional networks: An fMRI study with children and adults. Neurolmage, 28(2), 429-439. https://doi. org/10.1016/i.neuroimage.2005.06.065

Lawrence, M. A., \& Klein, R. M. (2013). Isolating exogenous and endogenous modes of temporal attention. Journal of Experimental Psychology: General, 142(2), 560-572. https://doi.org/10.1037/a0029023

Lewis, F. C., Reeve, R. A., \& Johnson, K. A. (2018). A longitudinal analysis of attention networks in 6- to 11-year-old children. Child Neuropsychology, 24(2), 145-165. https://doi.org/10.1080/09297049.2016.1235145

Lin, C. C. H., Hsiao, C. K., \& Chen, W. J. (1999). Development of sustained attention assessed using the Continuous Performance Test among children 6-15 years of age. Journal of Abnormal Child Psychology, 27(5), 403-412. https://doi. org/10.1023/A:1021932119311

Mezzacappa, E. (2004). Alerting, orienting, and executive attention: Developmental properties and sociodemographic correlates in an epidemiological sample of young, urban children. Child Development, 75(5), 1373-1386. https://doi.org/10.1111/j.1467-8624.2004.00746.x

Mullane, J. C., Lawrence, M. A., Corkum, P. V., Klein, R. M., \& McLaughlin, E. N. (2016). The development of and interaction among alerting, orienting, and executive attention in children. Child Neuropsychology, 22(2), 155-176. https:// doi.org/10.1080/09297049.2014.981252

Ostatníková, D., Pastor, K., Putz, Z., Dohnányiová, M., Matašeje, A., \& Hampl, R. (2002). Salivary testosterone levels in preadolescent children. BMC Paediatrics, 2, Article 5. https://doi.org/10.1186/1471-2431-2-5

Petersen, A., Petersen, A. H., Bundesen, C., Vangkilde, S., \& Habekost, T. (2017). The effect of phasic auditory alerting on visual perception. Cognition, 165, 73-81. https://doi.org/10.1016/j.cognition.2017.04.004

Petersen, S. E., \& Posner, M. I. (2012). The attention system of the human brain: 20 years after. Annual Review of Neuroscience, 35(1), 73-89. https://doi. org/10.1146/annurev-neuro-062111-150525

Posner, M. I. (2008). Measuring alertness. Annals of the New York Academy of Sciences, 1129(1), 193-199. https://doi.org/10.1196/annals.1417.011

Posner, M. I. (2012). Imaging attention networks. Neurolmage, 61(2), 450-456. https://doi.org/10.1016/i.neuroimage.2011.12.040

Quaiser-Pohl, C., Jansen, P., Lehmann, J., \& Kudielka, B. M. (2016). Is there a relationship between the performance in a chronometric mental-rotations test and salivary testosterone and estradiol levels in children aged 9-14 years? Developmental Psychobiology, 58(1), 120-128. https://doi.org/10.1002/dev.21333

Querne, L., Vernier-Hauvette, M. P., \& Berquin, P. (2009). Development of phasic attention in children: Temporal analysis of alert during a detection task. Current Psychology Letters, 25(1), 1-18. https://doi.org/10.4000/cpl.4800

Rueda, M. R., Fan, J., McCandliss, B. D., Halparin, J. D., Gruber, D. B., Lercari, L. P., \& Posner, M. I. (2004). Development of attentional networks in childhood. Neuropsychologia, 42(8), 1029-1040. https://doi.org/10.1016/i. neuropsychologia.2003.12.012

Schuhfried, G. (2011). Reaction test: Manual. Vienna test system. Schuhfried.

Shalev, N., Humphreys, G., \& Demeyere, N. (2016). Assessing the temporal aspects of attention and its correlates in aging and chronic stroke patients. Neuropsychologia, 92, 59-68. https://doi.org/10.1016/..neuropsychologia.2016.08.001

Shieh, K. K., Kuo, W. M., \& Lai, C. J. (1997). Effects of duration of stimulus and variability of foreperiod on the identification of multidimensional stimuli. Perceptual and Motor Skills, 84(3_Suppl.), 1379-1393. https://doi.org/10.2466/ pms.1997.84.3c.1379

Sobeh, J., \& Spijkers, W. (2013). Development of neuropsychological functions of attention in two cultures: A cross-cultural study of attentional performances of Syrian and German children of pre-school and school age. European Journa of Developmental Psychology, 10(3), 318-336. https://doi.org/10.1080/17405629. 2012.674761

Stenbaek, D. S., Fisher, P. M., Budtz-Jørgensen, E., Pinborg, A., Hjordt, L. V., Jensen, P. S., Knudsen, G. M. \& Frokjaer, V. G. (2016). Sex hormone manipulation slows reaction time and increases labile mood in healthy women. Psychoneuroendocrinology, 68, 39-46. https://doi.org/10.1016/i.psyneuen.2016.02.023

Sturm, W., Longoni, F., Fimm, B., Dietrich, T., Weis, S., Kemna, S., Herzog, H., \& Willmes, K. (2004). Network for auditory intrinsic alertness: A PET study. Neuropsychologia, 42(5), 563-568. https://doi.org/10.1016/j.neuropsychologia.2003.11.004

Sturm, W., \& Willmes, K. (2001). On the functional neuroanatomy of intrinsic and phasic alertness. Neurolmage, 14(1), S76-S84. https://doi.org/10.1006/ nimg.2001.0839

Tremolada, M., Taverna, L., \& Bonichini, S. (2019). Which factors influence attentional functions? Attention assessed by KiTAP in 105 6-to-10-year-old children. Behavioral Sciences, 9(1), Article 7. https://doi.org/10.3390/bs9010007

Waszak, F., Li, S. C., \& Hommel, B. (2010). The development of attentional networks: Cross-sectional findings from a life span sample. Developmental Psychology, 46(2), 337-349. https://doi.org/10.1037/a0018541

Yao, Q., Zhou, G., Xu, M., Dai, J., Qian, Z., Cai, Z., Zhang, L., Tan, Y., \& Hu, R. (2019). Blood metal levels and serum testosterone concentrations in male and female children and adolescents: NHANES 2011-2012. PLOS ONE, 14(11), Article e0224892. https://doi.org/10.1371/journal.pone.0224892

Zimmermann, P., \& Fimm, B. (2002). Testbatterie zur Aufmerksamkeitsprüfung (TAP) [Test of attentional performance (TAP)]. Version 1.7. Psytest. 